\title{
Comment
}

Neuroepidemiology 2010;35:115-116

DOI: $10.1159 / 000314928$

\section{Mobile Phone Use and Brain Cancer: Is the Association Biased?}

Michael Kundi

Institute of Environmental Health, Center for Public Health, Medical University of Vienna, Vienna, Austria

In the past decade more than 20 epidemiological studies investigating the relationship between mobile phone use and brain tumors have been published. Several meta-analyses [1-4] concluded that for malignant brain tumors there is an indication of an increased risk from long-term use especially when use of a mobile phone on the same side of the head where the tumor developed (ipsilateral use) is considered.

An essential problem in case-control studies is the retrospective exposure assessment. Most of the studies were either from the Interphone consortium or the Hardell group from Sweden that applied different protocols for exposure assessment. In the Interphone studies most of the interviews were conducted as computer-assisted personal interviews, while the Hardell group used mailed questionnaires. Information regarding mobile phone use extracted by these methods was similar: duration of mobile phone use, number and duration of calls, use of headsets etc. and side of the head the phone was held during calls. The Hardell group found increased risks overall and especially increasing risk estimates with prolonged duration since the first use of a mobile phone. Risk further increased if ipsilateral exposure was considered. Also the Interphone trial found an increased risk for longterm ipsilateral mobile phone use [5], however, estimates were lower than reported by the Hardell group.

These results provoked a controversy concerning potential reporting bias [6]. It has been argued that there is over-reporting of ipsilateral mobile phone use in cases because of reduced risk estimates for the contralateral side. However, this was an artifact of the estimation procedure. No contralaterally reduced risk was found when the analyses were applied correctly [5].

Validation studies carried out within the Interphone trial comparing self-reports with data from network operators $[7,8]$ revealed no differential bias for short-term recall but a possible overestimation in cases for mobile phone use in the more distant past, which, however, relied on data from one country only. Overall, these validation studies did not support the assumption of a distorting recall bias.

The results of the Hardell group, not only for malignant brain tumors but also for meningioma and acoustic neuroma, indicate distinctly higher risks as compared to those of the Interphone group, although the difference was less pronounced for long-term and ipsilateral use. It has been speculated that this discrepancy might be due to a distorting bias in the Hardell studies $[9,10]$. For example, it has been stressed that Hardell et al. [11] reported twice as many diagnoses of previous cancers among brain tumor cases than among controls, which could have influenced risk estimates. While it is a well-known fact that cancer survivors have an increased risk for secondary cancer and the mentioned discrepancy between cases and controls must have occurred in all other studies as well, the Hardell group was the first to report previous diagnoses; they demonstrated that if cases and controls with previous cancer affected results at all then by reducing risk estimates. The questionnaire method has also been mentioned as a source of recall bias, while other researchers [2] highlighted exposure assessment blinded to case status by the Hardell group. Indeed, interview and questionnaire methods have their pros and cons: interviews may introduce a bias from the expectations of the interviewer and the interaction between interviewee and interviewer; interviews are more demanding than answering a questionnaire at home where it is possible to check telephone bills or to inspect contracts with network providers to verify data. For these reasons, the questionnaire method seems to be superior to the interview technique. However, personal interviews also have their advantages: data can be immediately checked for errors and discrepancies, and the interviewer can assist in recalling inquired items. Due to memory problems and a reduced cognitive capacity data collection soon after surgery seems to be prone to recall bias. Such problems are less pronounced if data are collected by questionnaire and patients can go through the items at their own pace. A disadvantage is, however, that lag time between diagnosis and data collection is longer and many patients may die before contacted. In their studies of malignant brain tumors Hardell et al. [12] excluded $27 \%$ of cases for this reason while in the Interphone study [5] the corresponding figure was $18 \%$ only, which can be attributed to earlier data collection with more than $40 \%$ of those interviewed still being in hospital. The Hardell studies have particularly been criticized for excluding deceased patients (although this was the case in the Interphone studies as well). Should mobile phone use enhance tumor growth rate and speed up progression it is possible that it also increases case fatality rate. In this case leaving out dead and terminally ill cases would bias risk estimates towards zero. A bias in the other direction, i.e. a spuriously increased risk, is not possible.

There is an ongoing discussion in epidemiology how to handle the high case fatality rate of some diseases and the possible biases introduced by either leaving out these cases or collecting retrospective information from other sources (e.g. by proxy interviews). It was proposed to match deceased patients to deceased controls [13] in order to avoid bias by differential exposure assessment. Exactly this was done by the Hardell group using two types of matched controls: individuals that died from cancers other than brain cancer and individuals that died from non-malignant diseases. For cases as well as both types of controls the same ques-

\section{KARGER}

Fax +41613061234 E-Mail karger@karger.ch www.karger.com
(2) 2010 S. Karger AG, Basel

0251-5350/10/0352-0115\$26.00/0 
tionnaire-based data collection method was applied. Results are presented in this volume of Neuroepidemiology [14]. By this approach two essential problems were solved: first, the magnitude of bias introduced by leaving out deceased patients can be assessed, and second, the influence of attribution bias on risk estimates can be determined. The first is immediately evident and results indicate that bias from leaving out deceased patients is small (considering that non-differential exposure misclassification from relying on proxy information reduces risk estimates). The second follows from consideration of the origin of reporting bias: it has been argued that patients with a disease such as cancer seek explanation for the cause of their illness and might blame the mobile phone. If this is the case they might overrate amount and duration of use. It may be argued that relatives are less fallible in this respect. But most importantly, if there is also in relatives such a tendency of overestimation, it must be more pronounced in relatives of individuals that died from cancer than from other causes. Consequently, if there is a bias that leads to spuriously increased risk estimates, this would manifest itself by distinctly higher odds ratios using non-cancer controls. Hardell's group showed that this is not the case. There is no significant difference between odds ratios using cancer or non-cancer controls. For either sets of controls odds ratios are elevated for intensive use of a mobile phone.

For the time being, evidence collected in epidemiological studies points rather to an underestimation of risk from mobile phone use and less bias in the Hardell studies as compared to those of the Interphone group that are affected by selection and possibly also by misclassification bias.

\section{References}

$\checkmark 1$ Kundi M: The controversy about a possible relationship between mobile phone use and cancer. Environ Health Perspect 2009;117:316-324.

-2 Myung SK, Ju W, McDonnell DD, Lee YJ, Kazinets G, Cheng CT, Moskowitz JM: Mobile phone use and risk of tumors: a meta-analysis. J Clin Oncol 2009;27:5565-5572.

3 Khurana VG, Teo C, Kundi M, Hardell L, Carlberg M: Cell phones and brain tumors: a review including the long-term epidemiologic data. Surg Neurol 2009;72:205-214.

4 Hardell L, Carlberg M, Hansson Mild K: Epidemiological evidence for an association between use of wireless phones and tumor diseases. Pathophysiology 2009;16:113-122.

5 Lahkola A, Auvinen A, Raitanen J, Schoemaker MJ, Christensen HC, Feychting M, Johansen C, Klaeboe L, Lönn S, Swerdlow AJ, Tynes T, Salminen T: Mobile phone use and risk of glioma in 5 North European countries. Int J Cancer 2007;120:1769-1775.
6 Schüz J: Lost in laterality: interpreting 'preferred side of the head during mobile phone use and risk of brain tumour' associations. Scand J Public Health 2009;37:664-667.

7 Vrijheid M, Armstrong BK, Bédard D, Brown J, Deltour I, Iavarone I, Krewski D, Lagorio S, Moore S, Richardson L, Giles GG, McBride M, Parent ME, Siemiatycki J, Cardis E: Recall bias in the assessment of exposure to mobile phones. J Expo Sci Environ Epidemiol 2009;19:369381.

8 Vrijheid M, Cardis E, Armstrong BK, Auvinen A, Berg G, Blaasaas KG, Brown J, Carroll M, Chetrit A, Christensen HC, Deltour I, Feychting M, Giles GG, Hepworth SJ, Hours M, Iavarone I, Johansen C, Klaeboe L, Kurttio P, Lagorio S, Lönn S, McKinney PA, Montestrucq L, Parslow RC, Richardson L, Sadetzki S, Salminen T, Schüz J, Tynes T, Woodward A: Interphone Study Group. Validation of short term recall of mobile phone use for the Interphone study. Occup Environ Med 2006;63:237243.

9 Boice JD, McLaughlin JK: Epidemiologic studies of cellular telephones and cancer risk: a review. SSI Publication 2002:16. Accessed at http://www.stralsakerhetsmyndigheten.se/Publikationer/Rapport/ Stralskydd/2002/200216/.

10 Health Council of the Netherlands. ELF Electromagnetic Fields Committee. Electromagnetic Fields: Annual Update 2003. The Hague, Health Council of the Netherlands, 2004, No 2004/01.

- 11 Hardell L, Hallquist A, Hansson Mild K, Carlberg M, Påhlson A, Lilja A: Cellular and cordless telephones and the risk for brain tumours. Eur J Cancer Prev 2002;11:377-386.

12 Hardell L, Carlberg M, Hansson Mild K: Pooled analysis of two casecontrol studies on use of cellular and cordless telephones and the risk for malignant brain tumours diagnosed in 1997-2003. Int Arch Occup Environ Health 2006;79:630-639.

13 Gordis L: Should dead cases be matched to dead controls? Am J Epidemiol 1982;115:1-5.

14 Hardell L, Carlberg M, Hansson Mild K: Mobile phone use and the risk for malignant brain tumors: a case-control study on deceased cases and controls. Neuroepidemiology 2010;35:109-114.

Michael Kundi

Medical University of Vienna

Kinderspitalgasse 15

AT-1095 Vienna (Austria)

Tel. +4314277 64700, Fax +43142779647

E-Mail Michael.Kundi@univie.ac.at 